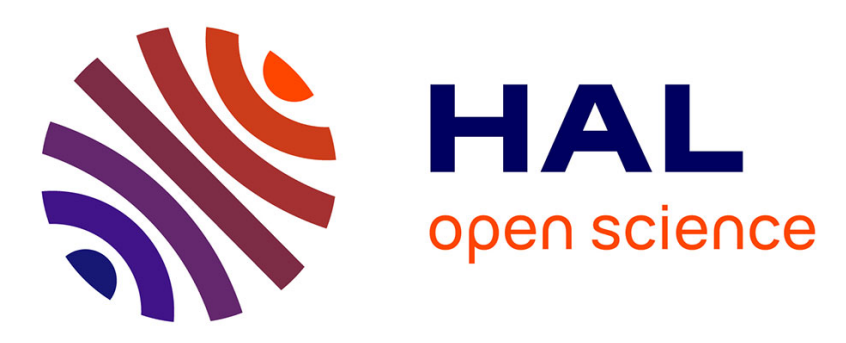

\title{
Monitoring of Hip and Knee Joint Angles Using a Single Inertial Measurement Unit During Lower Limb Rehabilitation
}

\author{
Vincent Bonnet, Vladimir Joukov, Dana Kulić, Philippe Fraisse, Nacim \\ Ramdani, Gentiane Venture
}

\section{To cite this version:}

Vincent Bonnet, Vladimir Joukov, Dana Kulić, Philippe Fraisse, Nacim Ramdani, et al.. Monitoring of Hip and Knee Joint Angles Using a Single Inertial Measurement Unit During Lower Limb Rehabilitation. IEEE Sensors Journal, 2016, 16 (6), pp.1557-1564. 10.1109/JSEN.2015.2503765 . lirmm-01396647

\section{HAL Id: lirmm-01396647 https://hal-lirmm.ccsd.cnrs.fr/lirmm-01396647}

Submitted on 14 Nov 2016

HAL is a multi-disciplinary open access archive for the deposit and dissemination of scientific research documents, whether they are published or not. The documents may come from teaching and research institutions in France or abroad, or from public or private research centers.
L'archive ouverte pluridisciplinaire HAL, est destinée au dépôt et à la diffusion de documents scientifiques de niveau recherche, publiés ou non, émanant des établissements d'enseignement et de recherche français ou étrangers, des laboratoires publics ou privés. 


\title{
Monitoring of Hip and Knee Joint Angles Using a Single Inertial Measurement Unit During Lower Limb Rehabilitation
}

\author{
Vincent Bonnet, Vladimir Joukov, Dana Kulić, Member, IEEE, Philippe Fraisse, Member, IEEE, \\ Nacim Ramdani, and Gentiane Venture, Member, IEEE
}

\begin{abstract}
This paper investigated the possibility of estimating 3D lower limb joint kinematics during five popular rehabilitation exercises of the hip and knee joints based on the data collected from a single inertial measurement unit located on the shank. The leg was modeled as a four-degree-of-freedom serial chain, and the relevant joint angles were represented by Fourier series. A least square approach based on the minimization of the difference between the measured and estimated 3D linear accelerations and angular velocities was used to solve the related analytical problem. The approach was validated on ten healthy young volunteers (ten trials each), comparing the proposed approach with the measurements collected through a stereophotogrammetric system. The average root mean square differences between the estimated joint angles and those reconstructed with the stereophotogrammetric system were inferior than $3.2^{\circ}$ with correlation coefficients higher than $\mathbf{0 . 8 5}$.
\end{abstract}

Index Terms - Inertial measurement unit, motion analysis, hip and knee rehabilitation.

\section{INTRODUCTION}

$\mathbf{T}$ HE monitoring of any rehabilitation program is desirable to ensure the correct execution of the exercise by the patient and also to quantify the progress toward the recovery of muscle strength, endurance, and increase in the range of motion. Monitored rehabilitation exercises have been shown to be more effective than practice without feedback [1], [2]. Hip abduction/adduction, hip circumduction, and hip and knee flexion/extension are among the most popular and most effective rehabilitation exercises for improving lower-limb muscle

Manuscript received October 7, 2015; accepted November 10, 2015. Date of publication December 10, 2015; date of current version February 8, 2016 This work was supported by the Japanese Society for Promotion of Science under Grant 13559. The work of V. Joukov and D. Kulić was supported in part by the Natural Sciences and Engineering Research Council of Canada and in part by the University of Waterloo Research Ethics Committee, under Grant ORE\#18889. The associate editor coordinating the review of this paper and approving it for publication was Prof. Zeljko Ignjatovicc.

V. Bonnet and G. Venture are with the Department of Mechanical Systems Engineering, Tokyo University of Agriculture and Technology, Tokyo 183-0057, Japan (e-mail: bonnet.vincent@gmail.com).

V. Joukov and D. Kulić are with the Department of Electrical and Computer Engineering, University of Waterloo, Waterloo, ON N2L 3G1, Canada (e-mail: vjoukov@uwaterloo.ca; dana.kulic@uwaterloo.ca).

P. Fraisse is with the Laboratoire d'Informatique, de Robotique et de Microélectronique de Montpellier, Centre National de la Recherche Scientifique, Montpellier 2 University, Montpellier 34000, France (e-mail: fraisse@lirmm.fr).

N. Ramdani is with PRISME, University of Orleans, Bourges 38 818, France (e-mail: nacim.ramdani@univ-orleans.fr).

Digital Object Identifier 10.1109/JSEN.2015.2503765 function and treating or preventing osteoarthritis [3]. When patients perform these prescribed exercises at home, both treatment time and cost decrease [4]. However, it is important that at-home exercises are performed correctly to ensure the benefit of the rehabilitation regimen. Even when exercises are performed under supervision, often clinicians and physiotherapists use only visual, and thus subjective, observations of the patient's movement. For single joint motion, a goniometer [5] can be used. However, the goniometer must be carefully aligned with the joint center, it needs to be repositioned every time that the targeted joint is changed, and does not allow monitoring of multi-segmental motions. Multi-segmental motions are favoured by clinicians since they allow several muscles to be strengthened at the same time and also improve patients' general coordination [6]. A key requirement for clinical applications and in-home rehabilitation is the development of easy-to-use and low-cost instruments. Thanks to their ease of use, robust design, and low cost, Inertial Measurement Unit (IMU) sensors have gained popularity in rehabilitation and other motion tracking applications. Cappozzo [7], in his minimum measured input model framework, maximizes the quality and the amount of functional information that can be estimated from simplified experimental protocols and a minimal sensor set. Based on this framework, Bonnet et al. [6], [8] proposed methods to estimate planar lower-limb joint mechanics during a squat exercise exploiting data provided by a single IMU. For multi-segmental 3D motion analysis, most of the existing protocols require at least one IMU attached to each investigated body segment to estimate 3D joint kinematics [9], [10]. However, for clinical applications in a daily environment, it is desirable to minimize the number of IMUs [7]. In theory, it is possible to estimate the position and orientation of the sensor from IMU 3D angular velocities and linear accelerations, through the integration and double integration of these signals. Unfortunately, drift rapidly degrades the accuracy of position and orientation estimates obtained via time integration of raw signals [6]. Adaptive and recursive filters, such as the Kalman filter [11] or the Weighted Fourier Linear Combiner [8], have been used to cancel this nonlinear drift. Despite their real-time ability, the Kalman filter can only estimate 2D orientation accurately [11], and the Weighted Fourier Linear Combiner has been validated only for 3D orientation of pseudo-periodic motion [12] and 
is very sensitive to non-stationary signals [8]. A model based approach incorporating knowledge of the kinematic constraints of the investigated task can help to overcome the drift effect [10]. The model based approach allows the estimation of additional variables that are not directly measured by the IMU, such as multiple-degree-of-freedom joint angles from a single IMU [6], [8]. A monitored exercise routine requires on-going feedback to the patient by the physiotherapist or by an automated biofeedback system. A multi-degree-of-freedom, real-time animation displaying the patient's actual motion and the clinician's prescribed one is an intuitive interface [2]. However, especially for in-home rehabilitation, the type of feedback (visual, tactile or auditory) might be extremely simplified to reduce cost and maximise accessibility. Also, if the patient suffers from confounding conditions, it may be difficult to generate optimal desired joint trajectories. Providing only information about the maximal amplitude and motion pulsation of the targeted joint immediately after finishing a motion cycle may be sufficient. On the other hand, clinicians or physiotherapists might be interested in knowing posteriori the accurate joint trajectories to analyse possible compensations due to the pathology or to calculate spatio-temporal parameters during the rehabilitation session.

In this context, this study aims to develop an automated system that provides to patients and clinicians a pseudo-online estimate of 3D hip and knee joint angles, observed during five common lower-limb rehabilitation exercises. This study is an extension to $3 \mathrm{D}$ motions of the previous work of our group concerning the squat exercise [6]. The accuracy of the proposed method and its performance was assessed through experiments involving ten human subjects via a comparison with data from a stereophotogrammetric system.

\section{Methods}

\section{A. Data Segmentation}

Due to the pseudo-periodicity of the selected rehabilitation tasks, the motion can be easily segmented in real-time into motion cycles. To do so, at each sample of time the sum of the squared measured angular velocities is calculated and filtered using a zero-phase lag Butterworth low pass filter with a cut-off frequency of $2 \mathrm{~Hz}$. This very low-frequency filter was used only for segmentation, to remove any peaks due to sensor noise. The beginning of the task was detected using a threshold on this signal. The signal was time windowed with a period of $0.2 \mathrm{~s}$. To account for sensor noise, mean $\left(\mathrm{m}_{\mathrm{tr}}\right)$ and standard deviation $\left(\mathrm{sd}_{\mathrm{tr}}\right)$ threshold values of this signal were calculated over the window during a static phase. The mean of the signal and its standard deviation were computed within each window and the first instant when these values were higher than $1.5 \mathrm{~m}_{\text {tr }}$ was chosen as the beginning of the rehabilitation exercise. Subsequently, local peaks in this signal were identified from its derivative when exceeding 0.1 rad.s ${ }^{-2}$. Since the movement is cyclic, the IMU is moving back and forth. Consequently, at least two peaks can be observed; the second peak corresponding to the end of a cycle and the beginning of the next one is identified as the subsequent segment point. To avoid false positives, the mean and standard

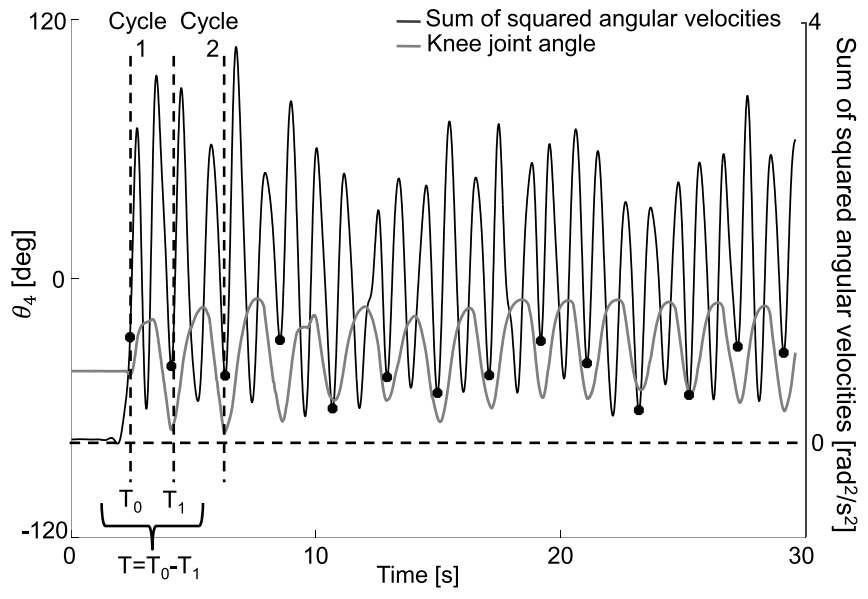

Fig. 1. Motion segmentation into motion cycles of duration T obtained during a knee flexion/extension exercise.

deviation and peak-to-peak temporal distance must exceed the following thresholds: $1.5 \mathrm{~m}_{\text {tr }}$ and $1.5 \mathrm{sd}_{\text {tr }}$ and $\mathrm{T}_{\text {tr }}=0.2 \mathrm{~s}$, respectively in order for the next segment point to be declared. Fig. 1 illustrates the signal segmentation and the knee angle during the first exercise, described in the following section.

\section{B. Mechanical Model}

The human leg was modelled as a 3D serial chain composed of three rigid segments (pelvis, thigh, and shank) and four rotational degrees-of-freedom (Fig.2). The origin of the serial chain, representing the global frame of reference $(\mathrm{G})$, is fixed in space and located at the pelvis level, while the end of the chain, point $U$, represents the center of the frame corresponding to the IMU sensor (u). The transformation matrices of the kinematic model are obtained following the modified Denavit-Hartenberg convention [13] and were calculated analytically. This minimizes the number of operations by creating intermediate variables and an analytical expression of the relationships that provide the acceleration vector of point $U$ represented in the IMU local frame, and the angular velocity vector of the shank as a function of the geometric parameters (the segment lengths) and of the kinematic variables of the model (joint angles and relevant time derivatives). The velocity ${ }^{G} \dot{\mathbf{P}}_{U}$ and acceleration vector ${ }^{G} \ddot{\mathbf{P}}_{\mathrm{U}}$ of point $\mathrm{U}$ were obtained by analytically differentiating and double differentiating the kinematic model:

$$
\begin{aligned}
{ }^{\mathrm{G}} \dot{\mathbf{P}}_{\mathrm{U}} & =\mathbf{J} \dot{\boldsymbol{\theta}} \\
{ }^{\mathrm{G}} \ddot{\mathbf{P}}_{\mathrm{U}} & =\dot{\mathbf{J}} \dot{\boldsymbol{\theta}}+\mathbf{J} \ddot{\boldsymbol{\theta}}
\end{aligned}
$$

where $\mathbf{J}$ is the time derivative of the Jacobian matrix $\mathbf{J}$ of the model described in Fig. 2, and the vectors $\dot{\boldsymbol{\theta}}$ and $\ddot{\boldsymbol{\theta}}$ denote the angular velocities and accelerations of the joints, respectively. The acceleration vector was represented in the IMU local frame $\left({ }^{\mathrm{u}} \ddot{\mathbf{P}}_{\mathrm{U}}={ }^{G} \boldsymbol{T}_{u}{ }^{\prime}{ }^{\mathrm{G}} \ddot{\mathbf{P}}_{\mathrm{U}}\right)$ via the inverse of the transformation matrix describing the IMU pose in the global frame of reference. 


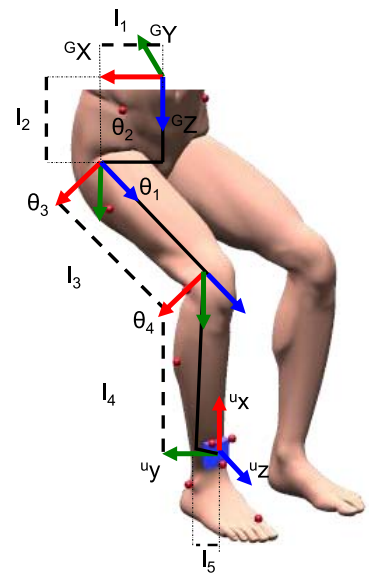

Fig. 2. Four-degree-of-freedom biomechanical model of the leg system. Point $\mathrm{U}\left({ }^{u} \mathrm{X},{ }^{u} \mathrm{Y},{ }^{u} \mathrm{Z}\right)$ represents the location of the IMU. The global frame ${ }^{G} \mathrm{X}^{G} \mathrm{Y}^{G} \mathrm{Z}$ is the global system of reference.

\section{Joint Angle Analytical Representation}

Since rehabilitation tasks are typically pseudo-periodic, $N$-harmonic Fourier series were used to model each joint motion:

$$
\theta_{j}(t)=a_{j 0}+\sum_{k=1}^{N}\left(a_{j k} \cos (k \omega t)+b_{j k} \sin (k \omega t)\right)
$$

where $j=1,2,3,4$ indicates the hip rotation, abduction and flexion and the knee flexion joints, respectively, and $\omega$ is the main frequency of the motion.

The analytical Fourier representation is convenient since it ensures that angular positions and velocities are continuously differentiable functions. The minimal value of $N$ was set as a trade-off between accuracy and computation time.

\section{Pseudo-Online Least-Squares Identification Algorithm}

The least-squares identification algorithm aimed to find the Fourier series coefficients $a, b$ and $\omega$, used to represent joint angles (eq.2) that minimize a cost function $C$. This cost function minimises differences between the estimated angular velocities and linear accelerations and their corresponding IMU measures and imposes a set of equality and inequality constraints. Inequality constraints were used to account for physiological limitations in the joint range of motion. Equality constraints were required to respect the initial measured joint angle $\left(\theta_{m j}(0)\right)$ positions. The proposed method might be used by the patient for self-correction while performing the rehabilitation exercise. Consequently, it is important to provide an estimate of joint angles in the minimal amount of computational time once a cycle has been executed. Solving the above mentioned non-linear constrained least-square identification problem using a gradient based optimisation method strictly satisfying the constraints, as proposed by Bonnet et al. [6], will require too much computational time. For this reason, the popular Levenberg-Marquadt algorithm [14], which is based on a gradient descent method and on the Gauss-Newton algorithm, was used. Since the Levenberg-Marquardt algorithm acts similarly to a gradient-descent method when the parameters are far from their optimal values, and relies more on the
Gauss-Newton method, which assumes that the least squares function is locally quadratic, when the parameters are close to the optimum, an appropriate choice of initial conditions will significantly reduce the computational time. The GaussNewton method typically converges much faster than gradientdescent methods [14]. However, the Levenberg-Marquadt does not handle constraints. To overcome this limitation a $\log$ barrier function expressed directly in the cost function [15] was chosen to handle the non-linear inequalities:

$$
\phi_{j}=\left\{\begin{array}{l}
-\log \left(\boldsymbol{\theta}_{j l i m}-\theta_{j}\right) \text { for } \theta_{j} \leq \theta_{j l i m} \\
+\infty
\end{array}\right.
$$

where $\boldsymbol{\theta}_{\text {jlim }}$ are the minimal and maximal limitations of the $\mathrm{j}^{\text {th }}$ joint as in [6].

Thanks to the shape of the log function, which increases rapidly to infinity when the joint angles approach their boundaries, no specific weights were required in the final cost function $C$. Note that for the investigated tasks the joint trajectories are far from their limitations, except for the knee joint, making these constraints almost non-influential. The equality constraints were easy to satisfy and they do not require a very high accuracy since the variability with which the initial joint angles may be measured is of several degrees [16]. Consequently, equality constraints were set as additional points to fit in the least-square identification problem. This equality was weighted using the number of considered samples. The inverse kinematic problem is not redundant, i.e. there is only one possible kinematic solution within the joint limitation. Consequently, the algorithm appears to be relatively insensitive to the value of this weight.

Finally, the following cost function $C$ was to be solved:

$$
\begin{gathered}
C=\frac{1}{n} \sum_{i=1}^{n}\left(\left({ }^{u} \ddot{P}_{\mathrm{U} m}(i)-{ }^{u} \ddot{P}_{\mathrm{U}}(i)\right)^{2}+\left({ }^{u} \dot{P}_{\mathrm{U} m}(i)-{ }^{u} \dot{P}_{\mathrm{U}}(i)\right)^{2}\right. \\
\left.+\sum_{j=1}^{4} \frac{n}{10}\left(\theta_{0 j}-\theta_{j}(1)\right)^{2}+\phi_{j}\right)
\end{gathered}
$$

where $n$ is the number of samples in one motion cycle. $\theta_{j}(1)$ refers to the first sample of each cycle and the index $m$ to the IMU's measured quantities.

The cost function is composed of elements of different units and the magnitude of the signals along the different axes of the exercises can change greatly. However, since the proposed method should be able to detect incorrect motions along undesired joint angles all measured data are of importance. Optimizing all the weights of all angular velocity and all linear acceleration components for all the investigated trials appears to be cumbersome and might lead to subject specific solutions. Also giving more or less importance to one axis might reduce the ability of the proposed method to detect compensatory motions. Consequently, we have chosen not to use any weighting in the least-square identification process. The identification algorithm was run for the first motion cycle with the same initial conditions for all subjects and all trials, where $a_{j 0}=\theta_{\mathrm{j} 0}$ and $a_{j 1}$ was set to be equal to half of the maximal joint amplitudes, and all the other Fourier coefficients were set to 0 . The results of the identification of the Fourier series amplitude and phase coefficients, i.e. $a$ and $b$, of the first cycle were subsequently used as the initial conditions 


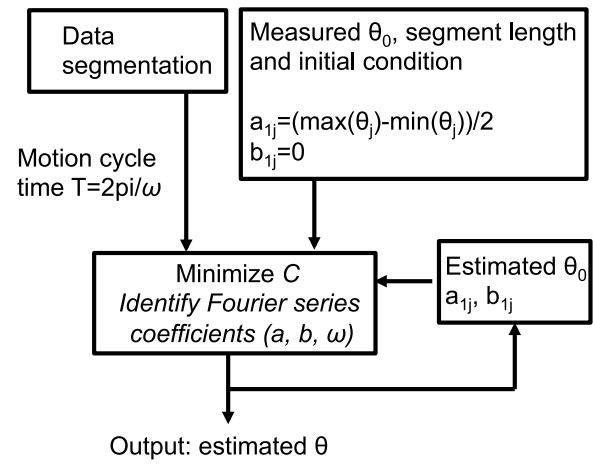

Fig. 3. Identification algorithm overview.

for the next cycle. The frequency initial condition value was calculated from the execution time of each cycle: $\omega=2 \pi / T$ where $T$ is the period of motion obtained from the data segmentation step. Fig. 3 presents the algorithm overview.

\section{EXPERIMENTAL VALIDATION}

\section{A. Experimental Setup}

Ten healthy volunteers $(9$ males and 1 females, age $=25 \pm 3$ years, mass $=77 \pm 14 \mathrm{~kg}$, height $=1.74 \pm 0.09 \mathrm{~m})$ participated in the study after signing an informed consent form. The model geometrical parameters, $l_{1}$ and $l_{2}$, used to calculate the constant rigid transformation from the global frame of reference to the hip joint center, were obtained by combining palpated locations of the right greater trochanter and superior iliac spines and regressive Bell's equations [17]. Other segment lengths were obtained from a palpation of common anatomical landmarks, between the great trochanter and the lateral condyle and the lateral malleolus, on the subject $\left(1_{3}, 1_{4}\right)$ and from the IMU CAD drawings $\left(1_{5}\right)$. The initial joint angles, $\theta(0)$, were measured using a goniometer while the volunteers were standing or sitting in their natural posture. An IMU ([18], $100 \mathrm{~Hz}$ ) was attached to the lower frontal part of the tibia and used to record 3D angular velocities and linear accelerations in the IMU frame. This location was chosen for its simplicity and to minimize measurement artefacts due to wobbling masses. A set of ten retro-reflective markers, placed on anatomical landmarks according to the commonly used Plug-in-Gait marker template (Vicon Motion Systems) was located on the right leg and pelvis of the subject as described in Fig. 4. In addition, a marker-cluster local frame was defined from three retro-reflective markers attached to the IMU sensor. A synchronized eight-camera stereophotogrammetric system (Motion Analysis, $100 \mathrm{~Hz}$ ) was used to record the $3 \mathrm{D}$ trajectories of the reflective markers. The stereophotogrammetric system was used only for validation purposes and is not required to use the proposed method in the clinical setting. Marker trajectories were used to drive the kinematic model described in Fig. 2 in a so-called global optimisation process [19] to estimate joint angles. The resultant joint angles were used as reference in validation of the proposed approach. Volunteers were asked to perform a series of five different motions as described in Fig. 4.

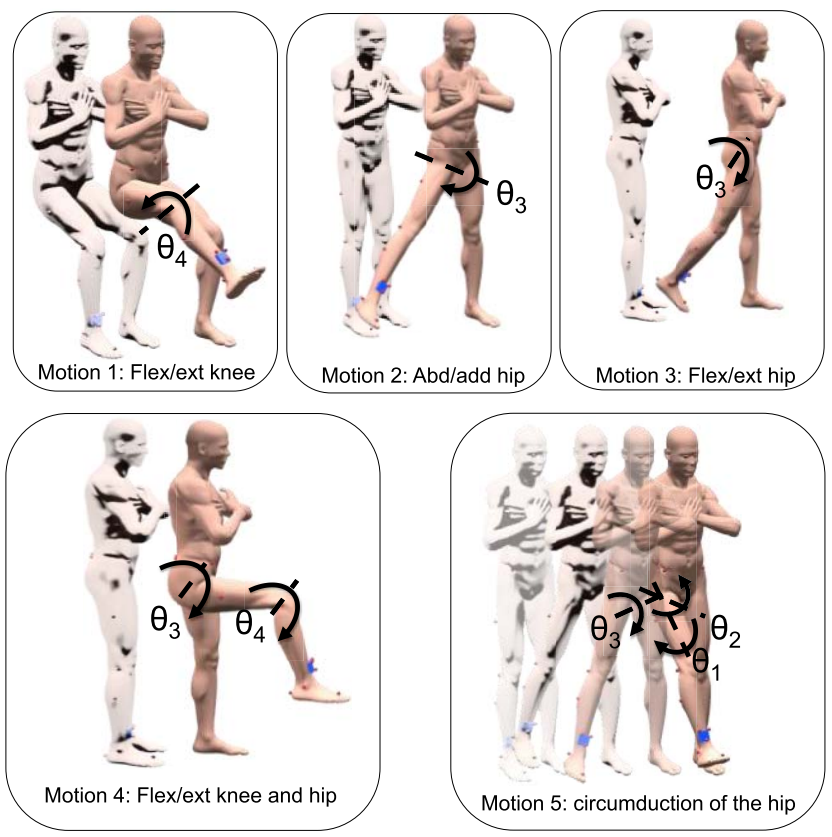

Fig. 4. Biomechanical description of the five investigated motions. Red dots represent the retro reflective markers used by the stereophotogrammetric system and the blue box represents the IMU sensor.

During the first motion, the subject was sitting on the edge of a table and was asked to perform maximal flexion/extension of the knee.

The second and third motions consisted of performing abduction/adduction and flexion/extension of the hip joint while standing on the contralateral leg and with fully extended knees, respectively. The fourth motion was a coordinated flexion/extension of the hip and knee joints while standing on one leg. Finally the last motion consisted of flexion/ extension, abduction/adduction and circumduction of the lower leg extremity with a fully extended knee. Subjects were standing on an elevated platform $10 \mathrm{~cm}$ off the ground and were asked to perform the exercises with minimal pelvis motion. They were allowed to hold on to a handrail with the contralateral hand to maintain balance. Each motion was repeated 10 times using a self-selected speed.

\section{B. Accuracy Analysis and Optimal Parameters Setting}

Instrument noise affecting the measured quantities was low-pass filtered at $5 \mathrm{~Hz}$ (Butterworth, 5th order). Hip and knee angles were estimated using both the proposed identification algorithm and the stereophotogrammetric data. The root mean square differences (RMSD), the Normalized RMSD, and the correlation coefficient $(r)$ between the investigated kinematic variables as estimated using the proposed method and as obtained using stereophotogrammetry were computed for the five hundred cycles. Two sensitivity analyses were conducted regarding the number of harmonics $N$ (eq. 2) and the influence of segment lengths on the estimate of joint angles. The optimal number of harmonics was determined by iteratively running the identification algorithm with $N$ ranging from 1 to 8 over the five trials of one randomly chosen subject. The RMSD between the IMU and stereophotogrammetry estimated joint 


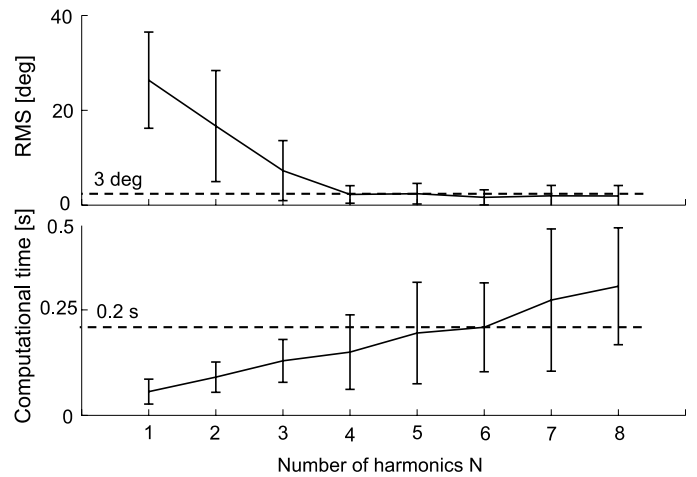

Fig. 5. Root mean square average difference between the angles of interest estimated using the proposed algorithm and via stereophotogrammetry for all five trials of a randomly chosen subject, as a function of the number of harmonics $\mathrm{N}$ (Top). Computational time required to identify the Fourier series coefficients as a function of the number of harmonics $\mathrm{N}$ that the latter algorithm uses to represent the joint angles (Bottom).

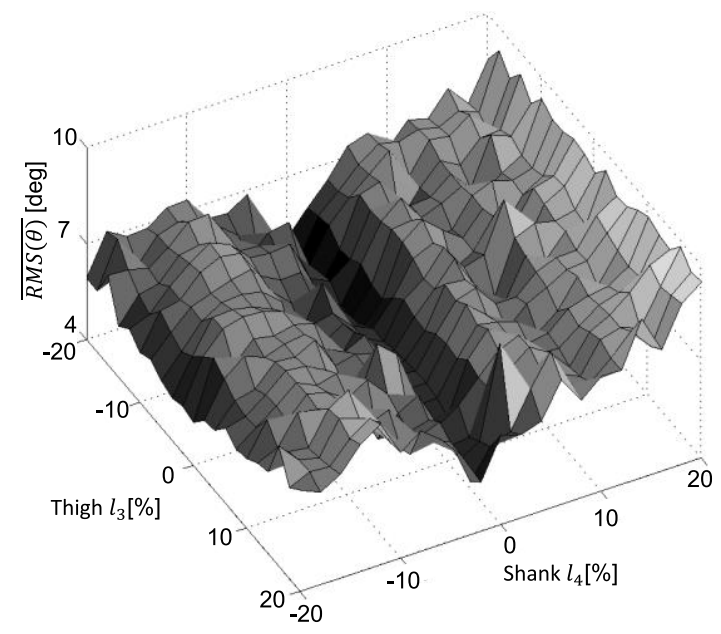

Fig. 6. Root mean square average difference between all the estimated angles using the proposed algorithm and via stereophotogrammetry for all five trials of a randomly chosen subject, as a function of the shank and thigh segment estimate accuracy.

angle values, and the computational time required to identify the Fourier series coefficients, were computed. The segment lengths influence the estimate of the linear accelerations since they are used in the Jacobian matrix $\boldsymbol{J}$ (eq.1). To analyse the influence of inaccurate segment length measurement, the average RMSD of all of the trials of a randomly chosen subject was calculated while varying the size of thigh $\left(l_{3}\right)$ and of shank $\left(1_{4}\right)$ segments by $\pm 20 \%$.

\section{RESUlts}

Fig. 5 summarizes the results obtained for the determination of the order $N$ of the Fourier series. For all investigated trials the average time to perform a cycle motion was $0.94 \pm 0.25 \mathrm{~s}$. Accordingly, a value inferior to $20 \%$ of this time was set as a threshold for the maximal calculation time available. A plateau in the accuracy of joint angle estimates of this subject is observable at approximately $3 \mathrm{deg}$. The first value allowing a drop below this threshold was $N=6$, with no evident improvement observed for the subsequent values of $N$.
TABLE I

Results of the Comparison of All the Angles as Obtained USING THE PROPOSED LEAST-SQUARES IDENTIFICATION TECHNIQUE AND STEREOPHOTOGRAMMETRY (GREY HIGHLIGHT THE ANGLES OF INTEREST - FIG. 4)

\begin{tabular}{|c|c|c|c|c|}
\hline & $\begin{array}{c}\text { Joi } \\
\text { nt } \\
\text { ang } \\
\text { le }\end{array}$ & $\begin{array}{l}\text { RMSD } \\
{[\mathrm{deg}]}\end{array}$ & $\begin{array}{c}\text { NRMS } \\
\%\end{array}$ & $r$ \\
\hline $\begin{array}{l}\text { Mot } 1 \\
\text { Knee Flex/ext }\end{array}$ & $\begin{array}{l}\theta_{1} \\
\theta_{2} \\
\theta_{3} \\
\theta_{4}\end{array}$ & $\begin{array}{l}3.5 \pm 1.4 \\
3.0 \pm 2.4 \\
3.8 \pm 2.1 \\
4.3 \pm 2.7\end{array}$ & $\begin{array}{l}33 \pm 13 \\
38 \pm 24 \\
32 \pm 21 \\
8 \pm 3\end{array}$ & $\begin{array}{l}0.74 \pm 0.20 \\
0.87 \pm 0.09 \\
0.80 \pm 0.14 \\
0.85 \pm 0.14\end{array}$ \\
\hline $\begin{array}{l}\text { Mot } 2 \\
\text { Hip Abd/add }\end{array}$ & $\begin{array}{l}\theta_{1} \\
\theta_{2} \\
\theta_{3} \\
\theta_{4}\end{array}$ & $\begin{array}{l}2.2 \pm 1.5 \\
2.2 \pm 1.0 \\
3.5 \pm 2.9 \\
2.2 \pm 1.8\end{array}$ & $\begin{array}{l}5 \pm 4 \\
9 \pm 7 \\
25 \pm 29 \\
11 \pm 7\end{array}$ & $\begin{array}{l}0.90 \pm 0.01 \\
0.90 \pm 0.12 \\
0.85 \pm 0.85 \\
0.92 \pm 0.10\end{array}$ \\
\hline $\begin{array}{l}\text { Mot } 3 \\
\text { Hip Flex/ext }\end{array}$ & $\begin{array}{l}\theta_{1} \\
\theta_{2} \\
\theta_{3} \\
\theta_{4}\end{array}$ & $\begin{array}{l}1.9 \pm 1.5 \\
1.4 \pm 1.2 \\
3.5 \pm 3.2 \\
1.4 \pm 0.9\end{array}$ & $\begin{array}{l}8 \pm 3 \\
7 \pm 6 \\
11 \pm 7 \\
8 \pm 6\end{array}$ & $\begin{array}{l}0.91 \pm 0.07 \\
0.93 \pm 0.07 \\
0.90 \pm 0.14 \\
0.92 \pm 0.11\end{array}$ \\
\hline $\begin{array}{l}\text { Mot } 4 \\
\text { Knee-hip }\end{array}$ & $\begin{array}{l}\theta_{1} \\
\theta_{2} \\
\theta_{3} \\
\theta_{4}\end{array}$ & $\begin{array}{l}2.7 \pm 1.4 \\
2.4 \pm 1.0 \\
3.6 \pm 2.4 \\
4.0 \pm 3.1\end{array}$ & $\begin{array}{l}7.5 \pm 3 \\
18 \pm 11 \\
9 \pm 6 \\
4 \pm 3\end{array}$ & $\begin{array}{l}0.82 \pm 0.10 \\
0.86 \pm 0.06 \\
0.97 \pm 0.03 \\
0.95 \pm 0.06\end{array}$ \\
\hline $\begin{array}{l}\text { Mot } 5 \\
\text { Hip } \\
\text { circumduction }\end{array}$ & $\begin{array}{l}\theta_{1} \\
\theta_{2} \\
\theta_{3} \\
\theta_{4}\end{array}$ & $\begin{array}{l}1.5 \pm 0.6 \\
3.6 \pm 1.1 \\
2.5 \pm 1.2 \\
2.6 \pm 1.2\end{array}$ & $\begin{array}{l}9 \pm 10 \\
17 \pm 12 \\
16 \pm 11 \\
27 \pm 19\end{array}$ & $\begin{array}{l}0.94 \pm 0.07 \\
0.90 \pm 0.07 \\
0.94 \pm 0.04 \\
0.90 \pm 0.07\end{array}$ \\
\hline
\end{tabular}

Fig. 6 shows the sensitivity analysis for the estimate of the segment lengths. As expected, the best results are obtained when the shank and thigh segment lengths are closest to their nominal values. Interestingly the thigh length does not have a large influence on the estimate of joint angles. On the other hand, one can see that an error of $\pm 10 \%$ of the shank length will degrade the joint angle estimate by almost a factor of two. However, it has to be noted that even in this case the RMSD will be acceptable (inferior to $8 \mathrm{deg}$ ). The pelvis peakto-peak 3D displacement and orientation change calculated from the pelvis markers were on average $14,33,12 \mathrm{~mm}$ and $3.3,3.7,3.6 \mathrm{deg}$, for all trials. These low values indicate that the assumption of a fixed pelvis base is suitable for the considered exercises. The average computational time for all the investigated trials was of $0.35 \pm 0.05 \mathrm{~s}$. The RMSD, NRMSD, and $r$ values, calculated over all 500 cycles between the estimated and all the measured angles are summarized in Table 1. The proposed algorithm was able to reconstruct the angles of the joint of interest with an RMSD of $3.2 \pm 1.2 \mathrm{deg}$. The average joint angle NRMSD was $10 \pm 4 \%$. The $r$ values were on average higher than 0.85 for all joints of interest. The NRMSD for some joint angles that are not specifically targeted during some motions, for example the hip rotation during flexion -extension of the knee, show large values with differences up to $40 \%$. However, these differences are due mainly to the fact that the motion amplitude at these joints is relatively small.

Fig. 7 shows the qualitative assessment of the similarity between the identification algorithm output and the 


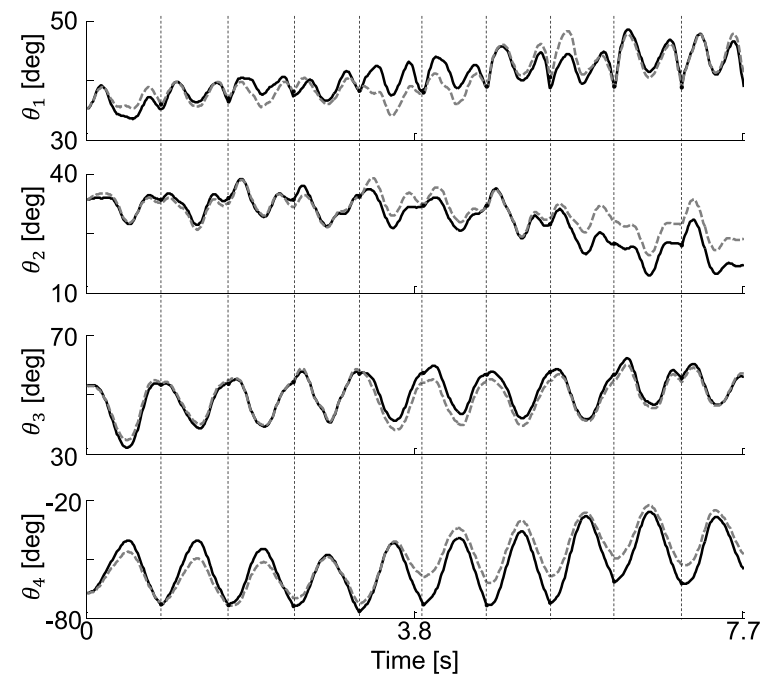

Fig. 7. Comparison between the values of the joint angles estimated from the IMU (dashed black lines) and those estimated from stereophotogrammetric data (solid black lines) for a coordinated flexion/extension of the knee and hip joints. The vertical dashed lines indicate the motion segmentation events.

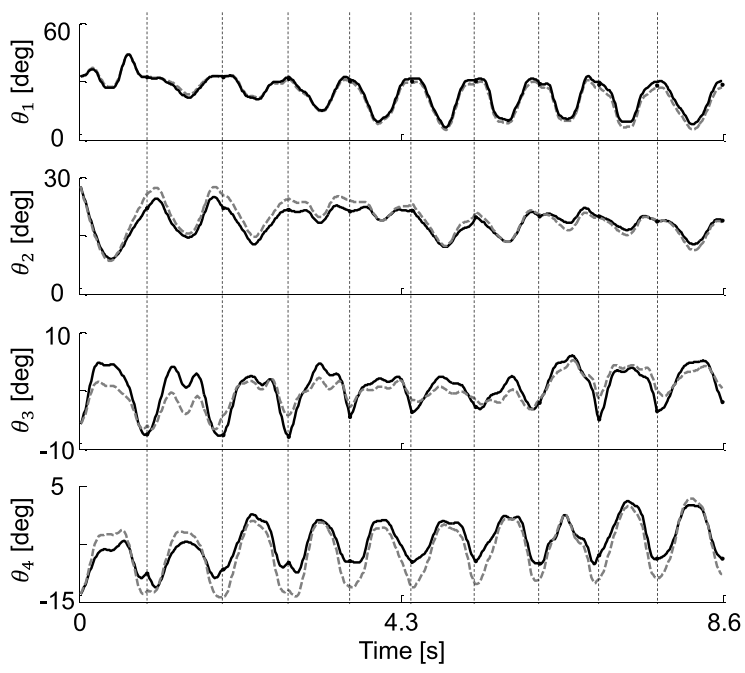

Fig. 8. Comparison between the values of the joint angles estimated from the IMU (dashed black lines) and those estimated from stereophotogrammetric data (solid black lines) for a circumduction of the hip joint. The vertical dashed lines indicate the motion segmentation events.

corresponding estimates from stereophotogrammetry for all the cycles of a trial corresponding to a coordinated flexion/extension of the knee and hip joint. The corresponding RMSD were: $\theta_{1}=2.9 \mathrm{deg}, \theta_{2}=3.4 \mathrm{deg}, \theta_{3}=2.1 \mathrm{deg}$ and $\theta_{4}=4.3 \mathrm{deg}$ and $r$ was above 0.87 . It is interesting to note that even though the task was supposed to be planar, non-negligible motions are observed on the hip rotation and adduction angles. This result highlights the importance of measuring 3D motion during exercise performance, and not only the motion of the targeted joint.

Fig. 8 presents another example of joint angle estimates for all the cycles of a trial corresponding to a circumduction of the hip. The corresponding RMSD were: $\theta_{1}=1.8 \mathrm{deg}$, $\theta_{2}=1.5 \mathrm{deg}, \theta_{3}=1.9 \mathrm{deg}$ and $\theta_{4}=2.7 \mathrm{deg}$ and $r$ was above 0.9. The identification algorithm was able to estimate accurately all joint angles even when displaying a relatively low level of periodicity such as for the hip flexion/extension angle, thanks to the high level of harmonics $(N=6)$.

\section{Discussion}

This study proposed a least-squares identification algorithm for the estimation of 3D lower-limb joint kinematic quantities during five common rehabilitation exercises targeting the hip and the knee joints; based on measurements from a single IMU located at the shank. The proposed approach is able to estimate the 3D joint angles with an average accuracy of $3.2 \mathrm{deg}$ and a correlation coefficient above 0.85 . These results are within the same range of error magnitude as reported in the literature. For example, when a mechanical device [20] was used to assess 3D knee joint angle estimates using IMU sensors, a RMS difference lower than 3 deg was found for the flexion angle. However, the studied range of motion was much larger than in our study and their orientation estimate algorithm was not detailed. Favre et al. [21] presented an RMS difference at the knee level of 1 degree for walking. However, this very good accuracy was obtained through multiple IMUs, after a calibration phase, and using a quaternion-based fusion algorithm. Finally, a model based approach, using constraints similar to the ones presented in this work, was used by Cooper et al. [22] with multiple IMU sensors for flexion/extension of the knee during walking with an RMS accuracy below one deg. As discussed by Brennan et al. [20], when comparing two motion analysis systems it is essential to use the same convention and metrics. Since the same four-degree-of-freedom mechanical model was used to estimate joint angles from the proposed method and from stereophotogrammetry, the discrepancies between the joint angles are solely an indicator of the difference between the results provided by the proposed method and the reference method. In the present study, the reported differences might be caused by IMU artefact motions creating undesired angular velocities and linear accelerations. During the minimisation of the cost function $C$ (eq.2) these measurement artefacts will then be fitted by the identification algorithm and will consequently degrade the joint angle estimate.

Another limitation of the proposed approach is the assumption of no pelvic motion. The quality of the results presented might be decreased if the exercises were performed with large pelvis movements. In order to cope with reasonably small involuntary pelvis motions the mechanical model could be extended with 3 additional rotations at the pelvis level. The cost function (eq. 4) could then be modified to estimate these additional degrees-of-freedom. However, in this case the problem would become under-determined. A reasonable assumption might be to minimise in the new cost function the amplitude of the pelvis motion. Nevertheless, a formal validation, assessing pelvis motion, should be performed for the specific pathologies or conditions that do not influence only knee and hip joints but the whole balance such as hemiplegia or Parkinson disease.

Initial joint position and segment lengths estimate will also have an influence on the joint angles estimate. The first one will propagate as a constant offset to all the subsequent motion cycles but will not influence the estimate of the amplitude 
between consecutive cycles. The estimate of the segment lengths will modify the joint angles estimate. However, if the estimated segment lengths are within $\pm 10 \%$ of their correct values, the RMSD will increase reasonably as shown in Fig. 6. For a segment of $40 \mathrm{~cm}$ this value is largely within the reported precision ( $5.5 \mathrm{~mm}$ for the medial condyle) of the detection of anatomical landmark positions with different examiners [23]. At contrary, the precision of the cycle detection will not have a large influence on the method accuracy since these events are used only to determine the initial value of the Fourier series pulsation that is subsequently optimised by the proposed method.

Despite the specific conditions of use, such as the minimisation of pelvis motion, to the best of our knowledge no other method proposes to accurately estimate the hip and knee joint angles using a single IMU, which is a real improvement in terms of cost and ease-of-use. From a computational point of view, the proposed approach based on Fourier series has the advantage of avoiding the use of error-prone numerical integration algorithms and was already fast enough $(0.35 \mathrm{~s}$ compared to $0.94 \mathrm{~s}$ average duration for one motion cycle) for developing a pseudo-online biofeedback system. The very low number of iterations required for the identification of Fourier series coefficients indicates that the problem is welldefined and converges even though the retained number of harmonics, set to $N=6$, leads to the identification of $6 \times 2 \times 4+4+4=56$ Fourier series coefficients. Additionally, the current implementation is in Matlab (Matworks) and a future $\mathrm{C}$ implementation and/or a proper analytical calculation of the cost function gradient should further reduce the time required for identification by at least a factor of 10 . However, the proposed approach requires that one motion cycle is fully executed before any results become available. The proposed approach requires a good initial estimate of the joint angles. These can be measured with a goniometer or during a specific constrained posture. However, an error in these estimates will propagate as a constant offset from one motion cycle to another and will degrade the absolute angle estimate but will not affect the estimate of the motion amplitude. The identification algorithm requires no subject specific parameter tuning, with the exception of the number of Fourier series harmonics and the measurement of initial joint position and segment lengths, and provides accurate results for all subjects and all investigated exercises. It can be concluded that the proposed method provides a robust estimate of kinematic quantities that have been shown to be of importance in the rehabilitation of hip and knee joints.

\section{REFERENCES}

[1] M. Friedrich, T. Cermak, and P. Maderbacher, "The effect of brochure use versus therapist teaching on patients performing therapeutic exercise and on changes in impairment status," Phys. Therapy, vol. 76, no. 10, pp. 1082-1088, 1996

[2] A. W. K. Lam, A. HajYasien, and D. Kulić, "Improving rehabilitation exercise performance through visual guidance," in Proc. 36th IEEE Int. Conf. Eng. Med. Bio. Soc., Chicago, IL, USA, Aug. 2014, pp. 1735-1738.

[3] M. E. Van Baar, J. Dekker, R. Oostendorp, D. Bijl, T. B. Voorn, and J. W. J. Bijlsma, "Effectiveness of exercise in patients with osteoarthritis of hip or knee: Nine months' follow up," Ann. Rheumatic Diseases, vol. 60, no. 12, pp. 1123-1130, 2001.
[4] A. Gonzalez, P. Fraisse, and M. Hayashibe, "Adaptive interface for personalized center of mass self-identification in home rehabilitation," IEEE Sensors J., vol. 15, no. 5, pp. 2814-2823, May 2015.

[5] J. Z. Edwards, K. A. Greene, R. S. Davis, M. W. Kovacik, D. A. Noe, and M. J. Askew, "Measuring flexion in knee arthroplasty patients," J. Arthroplasty, vol. 19, no. 3, pp. 369-372, 2004.

[6] V. Bonnet, C. Mazzà , P. Fraisse, and A. Cappozzo, "A least-squares identification algorithm for estimating squat exercise mechanics using a single inertial measurement unit," J. Biomech., vol. 45, no. 8 , pp. 1472-1477, 2012.

[7] A. Cappozzo, "Minimum measured-input models for the assessment of motor ability," J. Biomech, vol. 35, no. 4, pp. 437-446, 2002.

[8] V. Bonnet, C. Mazzà, P. Fraisse, and A. Cappozzo, "Real-time estimate of body kinematics during a planar squat task using a single inertial measurement unit," IEEE Trans. Biomed. Eng., vol. 60, no. 7, pp. 1920-1926, Jul. 2013.

[9] D. T.-P. Fong and Y.-Y. Chan, "The use of wearable inertial motion sensors in human lower limb biomechanics studies: A systematic review," Sensors, vol. 10, no. 12, pp. 11556-11565, 2010.

[10] J. F. S. Lin and D. Kulić, "Human pose recovery using wireless inertial measurement units," Physiol. Meas., vol. 33, no. 12, pp. 2099-2115, 2012.

[11] C. Mazzà, M. Donati, J. McCamley, P. Picerno, and A. Cappozzo, "An optimized Kalman filter for the estimate of trunk orientation from inertial sensors data during treadmill walking," Gait Posture, vol. 35, no. 1 , pp. $138-142,2012$.

[12] V. Bonnet, C. Mazzà , J. McCamley, and A. Cappozzo, "Use of weighted Fourier linear combiner filters to estimate lower trunk 3D orientation from gyroscope sensors data," J. Neuroeng. Rehabil., vol. 10, no. 1, p. $29,2013$.

[13] W. Khalil and E. Dombre, Modeling, Identification and Control of Robots. London, U.K.: Hermès Penton, 2002.

[14] D. W. Marquardt, "An algorithm for least-squares estimation of nonlinear parameters," J. Soc. Ind. Appl. Math., vol. 11, no. 2, pp. 431-441, 1963.

[15] J. Nocedal and S. Wright, Numerical Optimization. New York, NY, USA: Springer, 1999.

[16] U. D. Croce, A. Cappozzo, and D. C. Kerrigan, "Pelvis and lower limb anatomical landmark calibration precision and its propagation to bone geometry and joint angles," Med. Biol. Eng. Comput., vol. 37, no. 2, pp. 155-161, 1999.

[17] A. L. Bell, R. A. Brand, and D. R. Pedersen, "Prediction of hip joint centre location from external landmarks," Human Movement Sci., vol. 8 , no. 1, pp. 3-16, 1989.

[18] A. Burns et al., "SHIMMER-A wireless sensor platform for noninvasive biomedical research," IEEE Sensors J., vol. 10, no. 9, pp. $1527-1534$, Sep. 2010

[19] J. A. Reinbolt et al., "Determination of patient-specific multi-joint kinematic models through two-level optimization," J. Biomech., vol. 38, no. 3, pp. 621-626, 2005 .

[20] A. Brennan, J. Zhang, K. Deluzio, and Q. Li, "Quantification of inertial sensor-based 3D joint angle measurement accuracy using an instrumented gimbal," Gait Posture, vol. 34, no. 3, pp. 320-323, 2011.

[21] J. Favre, B. M. Jolles, R. Aissaoui, and K. Aminian, "Ambulatory measurement of 3D knee joint angle," J. Biomech., vol. 41, no. 5, pp. 1029-1035, 2008.

[22] G. Cooper et al., "Inertial sensor-based knee flexion/extension angle estimation," J. Biomech., vol. 42, no. 16, pp. 2678-2685, 2009.

[23] U. D. Croce, A. Leardini, L. Chiari, and A. Cappozzo, "Human movement analysis using stereophotogrammetry: Part 4: Assessment of anatomical landmark misplacement and its effects on joint kinematics," Gait Posture, vol. 21, pp. 226-237, no. 2, 2005.

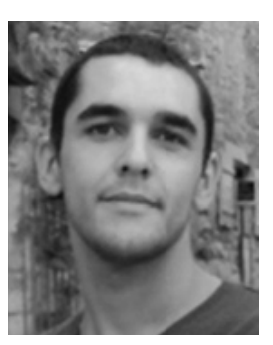

Vincent Bonnet received the B.E. degree in electrical engineering and the Ph.D. degree in automatics control and robotics from the Science University of Montpellier 2, in 2005 and 2009, respectively. From 2010 to 2012, he held a postdoctoral position in Rome, Italy, under the direction of Prof. A. Cappozzo. In 2013, he was a Teaching Assistant with the University of MontpellierEuromov Institute. He is with the Tokyo University of Agriculture and Technology, Tokyo, Japan. His research interests include various aspects of low-cost sensors, motion analysis, humanoid robotics, and system identification and control. 


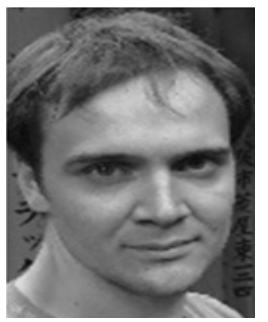

Vladimir Joukov received the B.E. and M.Sc. degrees in electrical and computer engineering from the University of Waterloo, where he is currently pursuing the Ph.D. degree with a focus on learning human motion control strategies and reproducing them on robots and active assistive devices. His research focused on estimating human pose during rehabilitation exercises using wearable inertial measurement units.

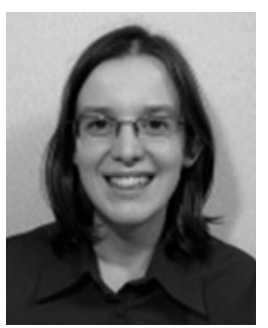

Dana Kulić received the B.A.Sc. and M.Eng. degrees in electromechanical engineering, and the Ph.D. degree in mechanical engineering from the University of British Columbia, Canada, in 1998 and 2005, respectively. From 2006 to 2009, she was a JSPS Post-Doctoral Fellow and Project Assistant Professor with the Nakamura Laboratory, University of Tokyo, Japan. She is currently an Associate Professor with the Electrical and Computer Engineering Department, University of Waterloo, Canada. In 2014, she received the Ontario's Early Researcher Award for her work on rehabilitation and human-robot interaction. Her research interests include human motion analysis, robot learning, humanoid robots, and human-machine interaction.

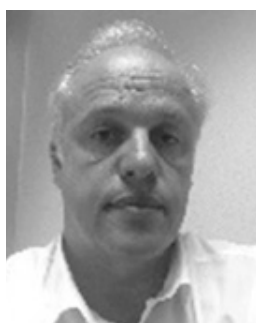

Philippe Fraisse received the master's degree in electrical engineering from the Ecole Normale Superieure de Cachan in 1988, and the Ph.D. degree in automatic control from University Montpellier 2, France, in 1994, with a focus on position/force control or two-arm robots. He is currently a Professor with the LIRMM, Laboratory, Montpellier 2 University, France. His research interests include human-robot interaction, humanoid robotics, robotics for rehabilitation, and mobile manipulators. He is involved in the Japanese-French Joint Laboratory for

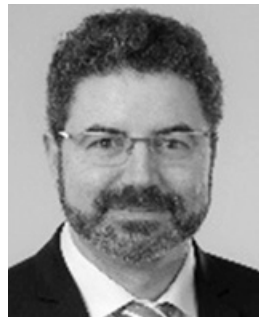

Nacim Ramdani received the Engineering degree from the Ecole Centrale de Paris, France, in 1990, and the Ph.D. degree from the University of ParisEst Creteil, France, in 1994. Since 2010, he has been a Full Professor with the University of Orleans. Until 2010, he was the Maitre de Conferences with University Paris-Est Creteil. $\mathrm{He}$ is the Co-Chair of the Workgroup on Set Computation Techniques within the French Research Group on Automatic Control and of the U.K. Working Group on Interval Methods. His current research interests revolve around developing robust numerical methods for the modeling and analysis of cyber-physical and autonomous systems in presence of uncertainty, and set membership estimation with applications to robotics, human-robot interaction, and healthcare.

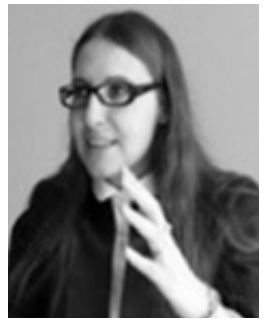

Gentiane Venture received the engineering degree in robotics and automation from the Ecole Centrale of Nantes, France, in 2000, the M.Sc. degree in robotics from the University of Nantes, France, and the Ph.D. degree from the University of Nantes, France, in 2003. In 2004, she joined the French Nuclear Agency, Paris, France. Later, she joined Prof. Yoshihiko Nakamura's Laboratory, University of Tokyo, Japan, with the support of the JSPS In 2009, she became an Associate Professor and starts a new lab with the Tokyo University of Agriculture and Technology, Japan. Her main research interests include non-verbal communication, human behavior understanding from motion, human body dynamics modeling, dynamics identification, control of robot for human/robot interaction, and human affect recognition.

Robotics, Tsukuba, Japan. 